\title{
Bulk GaAs room temperature radiation detectors
}

\author{
Douglas S. McGregor a, Glenn F. Knoll a, Yosef Eisen ${ }^{b}$ and Richard Brake ${ }^{c}$ \\ "Department of Nuclear Engineering. The Unitersity of Michigan, Ann Arbor, MI 48109-2100, USA \\ ' Soreq Nuclear Research Center, Israel Atomic Energy' Commission, Yaine 70600, Israel \\ ' Radiation Protection Group, Los Alamos National Laboratory, Los Alamos. NM 87545, USA
}

Bulk GaAs, a wide band gap semiconductor, shows potential as a room temperature radiation detector. Schottky diode detectors were fabricated from LEC bulk GaAs crystals. The basic construction of these diodes employed the use of a Ti/Au Schottky contact and a $\mathrm{Au} / \mathrm{Ge} / \mathrm{Ni}$ alloyed ohmic contact. Pulse height characteristics of these diodes indicate active regions of more than $10^{9} \mu \mathrm{m}$. Pulse height spectra were recorded from alpha particle irradiation of the Schottky contact surface resulting in a best energy resolution of $2.5 \%$ at $5.5 \mathrm{MeV}$. Low energy gamma rays measured under room temperature operating conditions resulted in photopeaks with $37 \%$ FWHM at $60 \mathrm{keV}$.

\section{Introduction}

In order for a semiconductor material to be useful as a radiation detector at room temperature, its bandgap value must be wide enough to inhibit excessive leakage current due to thermal carrier generation. As a practical matter, this requirement translates into a minimum bandgap energy of $1.4-1.5 \mathrm{eV}$ if relatively thick detcctors are to be fabricated [1,2]. Gallium arsenide (GaAs) is a wide bandgap semiconductor (1.42 $\mathrm{eV}$ at $25^{\circ} \mathrm{C}$ ) that has received attention as a candidate material for radiation detectors [3-5]. Its bandgap is sufficiently large to allow for roor temperature operation of bulk detectors and its averuge atomic number is equivalent to that of germanium, the most widely applied semiconductor material in gamma ray spectroscopy.

The investigation of $\mathrm{GaAs}$ as a detector material dates back to the late 1960s and early $1970 \mathrm{~s}[3,4]$. At that time, epitaxial growth was the only means available to produce high quality GaAs layers of sufficient thickness, generally limited to less than $100 \mu \mathrm{m}$. Since then, high quality semi-insulating GaAs material grown by the liquid encapsulated Czochralski (LEC) method has become widely available from a number of commercial suppliers. Compensation of background impurities has improved such that quoted resistivities $\left(>10^{7}\right.$ $\Omega \mathrm{cm})$ and carrier concentrations $\left(<10^{7} / \mathrm{cm}^{3}\right)$ approach theoretical intrinsic values. Compensation of high resistivity LEC GaAs material is accomplished by a balance between residual carbon shallow acceptor impurities with the native defect deep donor EL2 [6,7]. In this work, we report measured results from detec- tors fabricated from commercially available high purity semi-insulating LEC bulk GaAs crystals.

\section{Device fabrication}

Bulk LEC GaAs wafers were diced into $5.08 \mathrm{~mm} \times$ $5.08 \mathrm{~mm}$ pieces and polished to thicknesses of 45,100 and $250 \mu \mathrm{m}$. Previous results indicated the need for surface passivation to reduce leakage currents [8], thus $\mathrm{Si}_{3} \mathrm{~N}_{4}$ was plasma deposited onto each piece to provide necessary passivation. Photolithography was used to define $4.32 \mathrm{~mm}$ diameter circular regions which were then used to etch through the $\mathrm{Si}_{3} \mathrm{~N}_{4}$ to the bare GaAs. A series of alternating $\mathrm{Au}, \mathrm{Ge}$, and $\mathrm{Ni}$ layers were vacuum deposited onto the GaAs surfaces with weight ratios of $88 \%, 12 \%$, and $5 \%$, respectively. The contacts were annealed at $405^{\circ} \mathrm{C}$ to form ohmic contact to the GaAs substrates. Photolithography was then used to define and protect areas on the front surfaces $4.32 \mathrm{~mm}$ in diameter. The front side of the devices were ion implanted with protons at a dose of $10^{16} / \mathrm{cm}^{2}$ to decrease carrier lifetimes contributing to leakage on the outer edges of the substrate. Afterwards, $\mathrm{Si}_{3} \mathrm{~N}_{4}$ was deposited on the front surfaces, patterned, and etched as previously described. Each piece was bonded into a BN (boron nitride) collar with high resistivity potting epoxy. These BN collars were subsequently bonded into brass connector rings. A layer of $\mathrm{Ti} / \mathrm{Au}$ was vacuum deposited onto the bare GaAs surfaces to form a Schottky contact to the GaAs substrates and electrical conta:t to the front connector rings. A final Au layer was aeposited over the ohmic contact detector 
faces to form electrical contact to the back connector rings. A cross section diugram of a completed device can be seen in fig. 1 .

\section{j. Pevice operating characteristics}

The $C-I, I-l$, pulse height, and spectroscopic characteristics were observed for each device. I-V nteasurements indicated reverse bias breakdown voltages in cxcess of $60 \mathrm{~V}$ for $45 \mu \mathrm{m}$ thick detectors, and in excess of $140 \mathrm{~V}$ for the $100 \mu \mathrm{m}$ thick detectors. It was obsered that in all cases of reverse bias, the reverse current reached a maximum value and began to decrease with higher voltages. Reverse currents from the devices ianged in value from 27 to $500 \mathrm{nA}$. The detectors demonstrated rectifying behavior as shown in fig. 2. However, the ideality factors of the diodes as measured from the forward current were high, suggesting that the current was due to mechanisms other than thermionic emission. The turn-on voltage for forward bias dioudes was ubsened to increase with increasing detector thickn'ss.

Initial $C-1 \cdot$ measurements of the devices were taken with a modulating frequency of $1 \mathrm{MHz}$ resulting in no apparent change in capacitance with increasing reverse bias. Generally. the display of constant capacitance with changing bias voltage indicates full depletion. Howeser, $C-$ measurements of high resistivity materials can render erroneous results [9]. It is believed that the EL2 deep level donors in the material have lifetimes longer than or comparable to the period of the modulation frequency and conclusions as to the behavfor of the EL2 do:p donors and their effect on capacitance could not be made [9.10]. Consequently, conclusions as to the electric field distribution of these detectors could not be drawn through simple $C-V$ techniques.

Observations of the pulse height dependence on bias voltage were conducted to enhance understanding of the device characteristics. GaAs detectors $43 \mu \mathrm{m}$ thick and $105 \mu \mathrm{m}$ thick were irradiated by an ${ }^{241} \mathrm{Am}$

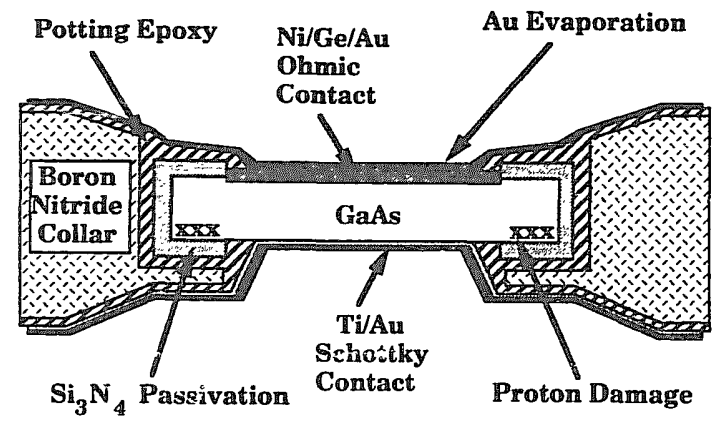

Fig. 1. Bulk GaAs detector device cross section.

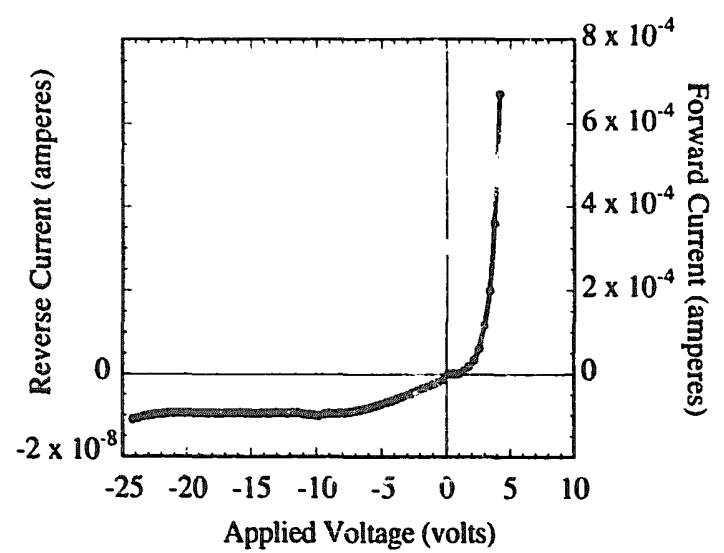

Fig. 2. $I-V$ characteristics of a $43 \mu \mathrm{m}$ thick GaAs Schottky barrier detector. The forward current increases much slower than that expected for an ideal diode. Reverse and forward currents are represented with different scales.

spectroscopic alpha particle source under vacuum from both the front (Schottky) contact and back (ohmic) contact. The range of a $5.5 \mathrm{MeV}$ alpha particle in GaAs is approximately $21 \mu \mathrm{m}$ [11]. Carrier generation is greatest near the end of the alpha particle track length allowing for the bulk of the electron-hole pairs to be created near the middle of the $43 \mu \mathrm{m}$ thick detector when irradiated from either side. The average pulse height produced by the alpha particles was measured as a function of bias voltage. For both thicknesses. the pulse height was observed to increase rapidly with increasing reverse bias when irradiated from the Schottky contact side. The pulse height saturated at a maximum value in all cases of front side irradiation, thus indicating extension of the detector active region beyond the alpha particle range (figs. 3 and 4). The voltage at which saturation occurred was similar for all thicknesses of detectors tested. Detector resolution improved with increasing bias voltage, indicating improved charge collection with increasing bias. The energy resolution was observed to reach a saturated value when the pulse height curve reached its $95 \%$ value (fig. 5). It is believed that the improving resolution is from increased charge collection resultant from the detector active region extending beyond the alpha particle range. Assuming that $4.2 \mathrm{eV}$ are necessary to creatc an electron-hole pair in GaAs $[3,4]$, preliminary measurements indicate near complete charge collection for the $45 \mu \mathrm{m}$ thick detectors as compared to the pulse height spectrum observed from a silicon surface barrier detector. The normalized pulse height characteristics of the GaAs detectors differ only slightly, indicating that the electric field distribution near the front contacts of both detectors are similar (fig. 6). In the hypothetical case in which a semicon- 


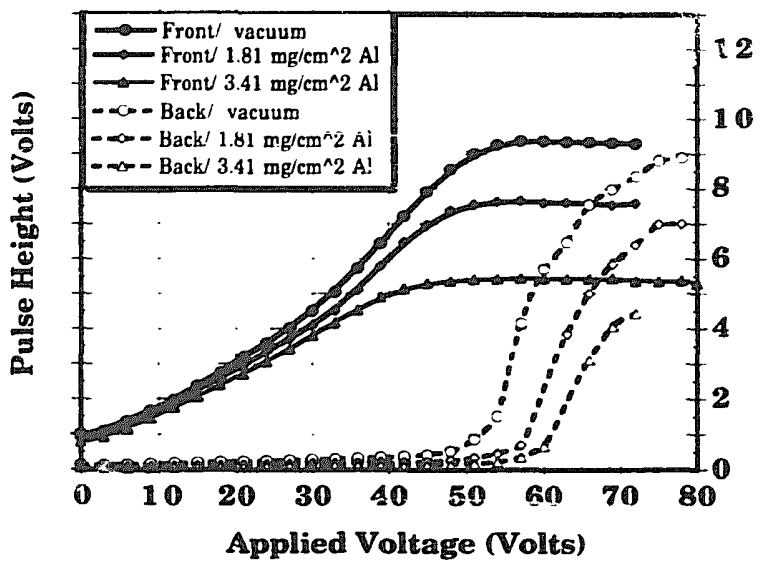

Fig. ? $\mathrm{M}$-asured pulse height as a function of bias voltage from a $4 ? \mu \mathrm{m}$ thick bulk GaAs detector. A spectroscopic grade ${ }^{2+1} \mathrm{Am}$ alpha particle source was used for the study. The source and detector were in a vacuum chamber and the pulse height recorded for conditions of no attenuation. attenuation with $1.81 \mathrm{gm} / \mathrm{cm}^{2} \mathrm{Al}$, and attenuation with $3.41 \mathrm{gm} / \mathrm{cm}^{2}$. 11 . Pulse heights were measured from the detectors as irradiated from the front (Schottky) contact and again from the back (ohmic) contact.

ducting material is intrinsic or well compensated, such as in zone refined or compensated $\mathrm{Ge}$, the expected result would be an electric field distribution across the device with a gradual slope. The electric field strength could then be estimated by simply dividing the applied voltage by the detector thickness. In such a case, the required voltage for the thicker detector should be 2.4 times greater than the thin detector to achieve the same electric field distribution, and consequently, a

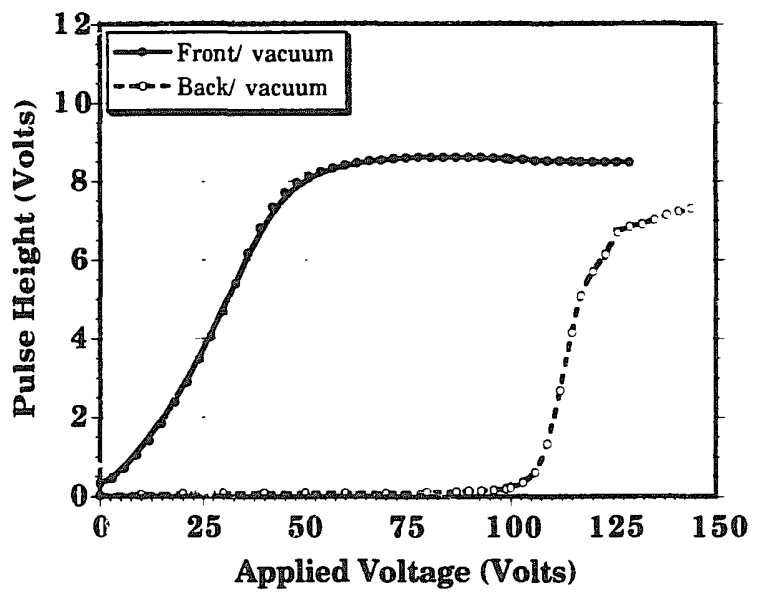

Fig. 4. Measured pulse height as a function of bias voltage from a $105 \mu \mathrm{m}$ thick bulk GaAs detector. A spectroscopic grade ${ }^{241} \mathrm{Am}$ alpha particle source was used and the source and detector were under vacuum. The pulse height response was recorded for conditions in which the front and back of the diodes were irradiated separately.

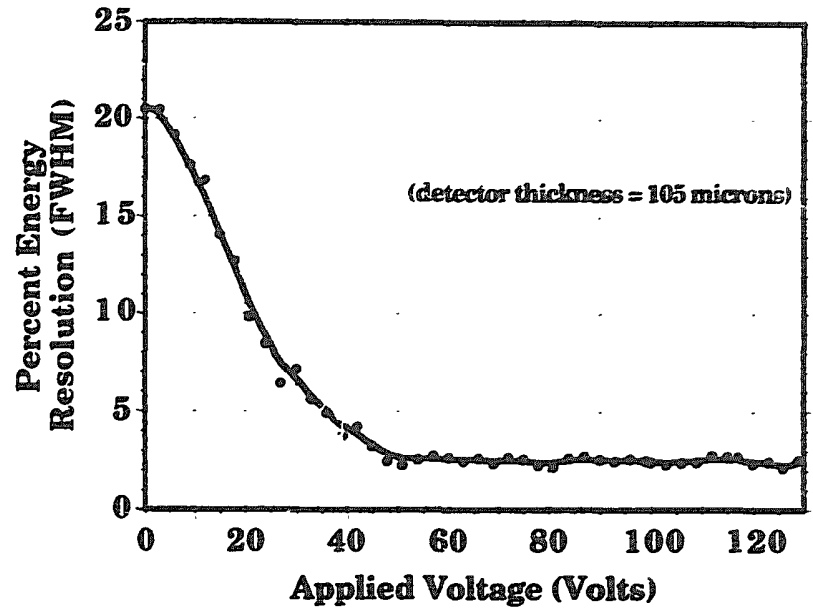

Fig. 5. Detector resolution as a function of reverse bias voltage for a $105 \mu \mathrm{m}$ thick GaAs detector.

similar pulse height curve. The fact that the pulse height observed is nearly independent of the detector thickness suggests an electric field behavior more typically found in doped semiconductor material rather than intrinsic or compensated material.

Under back side irradiation conditions. a very low pulse height was measured that slowly increased with bias voltage. With further applied voltage. a threshold voltage for dramatic increase in pulse height was observed indicating the point at which the active region reached the end of the alpha particle range (figs. 3 and 4). In all cases of back side irradiation, after the onset of pulse height increase a continuum of pulse heights appeared up to a maximum value. This continuum was

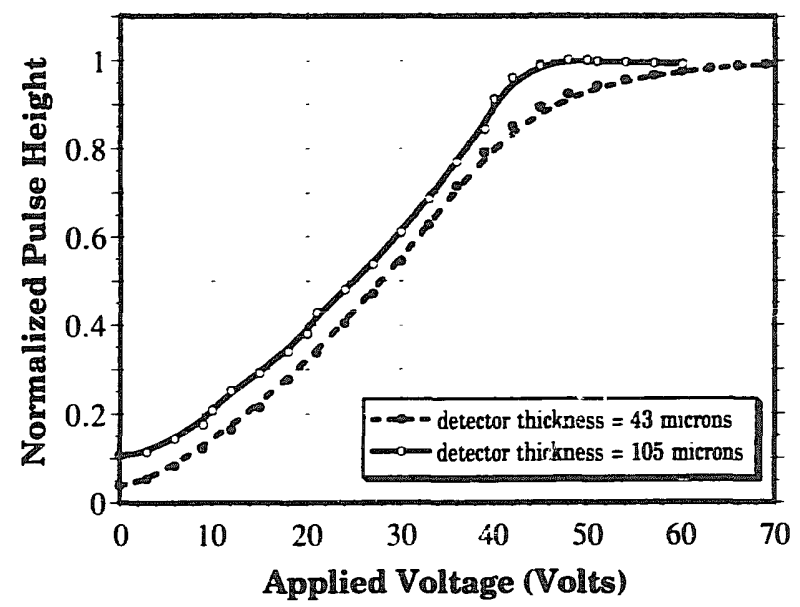

Fig. 6. Normalized pulse height curves comparing the output signal demonstrated by a $43 \mu \mathrm{m}$ thick detector and a $105 \mu \mathrm{m}$ thick detector when the Schottky contact was irradiated with alpha pariicles under vacuum. The similar characteristics indicate similar electric field distributions near the Schottky conlact for both devices. 
observed for a short range of voltages, after which a poor resolution full energy peak formed. In all cases when the source was unattenuated in vacuum, the saturated average pulse height voltage was less than the pulse height voltage observed from front side irradiation under similar conditions by approximately $8-$ 10r: depending on detector thickness. It is believed that the nature by which the ohmic contact: are made results in increased statistical fluctuations in energy deposition from back side irradiation. however, calculations of energy attenuation in the contact do not completely accoun for the lower saturated pulse height abserced. The centroid of the pulse height distribution continued to increase towards a saturated maximum value with increasing bias, thus indicating improvement in charge collection. Resolution degradation was obsarid to be worse in $100 \mu \mathrm{m}$ thick detectors than in $\$ 5 \mu \mathrm{m}$ thich deteciors. It is believed that poor hole collection due to trapping in the matcrial is the cause a inferior charge collection as compared to front side irradiation which iranslates into pulse height reduction and resolution degradation.

Aluminum attenuators were used to reduce the alpha particle energy and the pulse response was obsined from a $43 \mu \mathrm{m}$ thick detector. The range of the alpha particles in GaAs decreases with alpha energy. Thus, the woltage required to observe the onset of the vlrage plaicau should decrease with attenuator thickness wen the discetors are irradiated from the front. and the required threshold voltage for pulse height increase should increase with aticnuator thickness when irradiated from the back. From fig. 3. the unattenuated

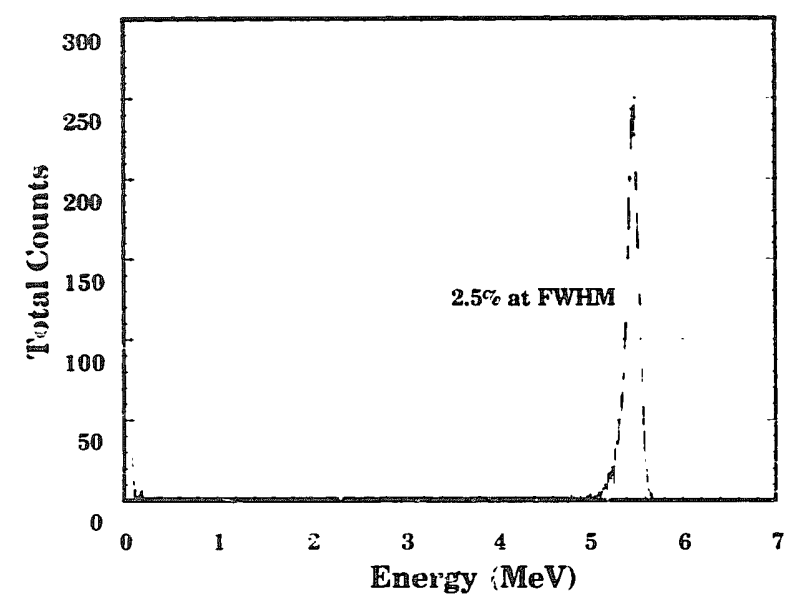

Fig. 7. Room temperature measurement of the disferential pulse height spectrum exhibited by a $105 \mu \mathrm{m}$ thick bulk GaAs detector inadiated while under vacuum with $5.5 \mathrm{MeV}$ alpha particles from ${ }^{2+1} \mathrm{Am}$. The detector resolution was measured to be $2.5 \%$ at FIVHM alpha particle pulse height spectrum reached the plateau voltage at approximately $57 \mathrm{~V}$ in a $43 \mu \mathrm{m}$ thick detector. With a $1.81 \mathrm{mg} / \mathrm{cm}^{2} \mathrm{Al}$ attenuator between the source and detector, the plateau begins at $51 \mathrm{~V}$. Similarly, the plateau voltage is $45 \mathrm{~V}$ when the alpha particles are attenuated by $3.41 \mathrm{mg} / \mathrm{cm}^{2}$ of Al. Back side irradiation of the detector resulted in the onset of pulse height increase at $49 \mathrm{~V}$ for unattenuated particles, $54 \mathrm{~V}$ for particles attenuated with $1.81 \mathrm{mg} / \mathrm{cm}^{2}$ $\mathrm{Al}$, and $57 \mathrm{~V}$ with $3.41 \mathrm{mg} / \mathrm{cm}^{2} \mathrm{Al}$.

Of interest is the fact that boundaries at which pulse height plateaus and thresholds occur do not match simplistic depletion approximation solutions. The dependence of active region width with applied voltage displays behavior similar to that expected from a nonuniformly doped semiconductor. The reason for such behavior may be a consequence of unionized EL2 donors under the condition of zero bias becoming ionized as voltage is applied. Applied reverse voltage can ionize EL.2 donor sites until the total concentration is fully ionized, which may translate to rapid decrease in electric ficld strength near the Schottky contact. Further discussion is provided in the appendix.

Noise levels of the GaAs detectors measured at room temperature ranged from 40 to $80 \mathrm{keV}$. Front side irradiation under vacuum and at room temperature resulted in a best energy resolution of $2.5 \%$ FWHM for $5.5 \mathrm{MeV}$ alpha particles (fig. 7). Room temperature measurements of $60 \mathrm{keV}$ gamma rays from an ${ }^{2+1} \mathrm{Am}$ source resulted in a best energy resoution of $37^{\circ}$, FWHM (fig. 8). Room temperature measurements of $122 \mathrm{keV}$ gamma rays from a ${ }^{57} \mathrm{Co}$ gamma ray source revealed a photopeak that suffered poor resolution. Measurements with gamma rays of higher energies resulted in the appearance of a continuum with no observable full energy peak.

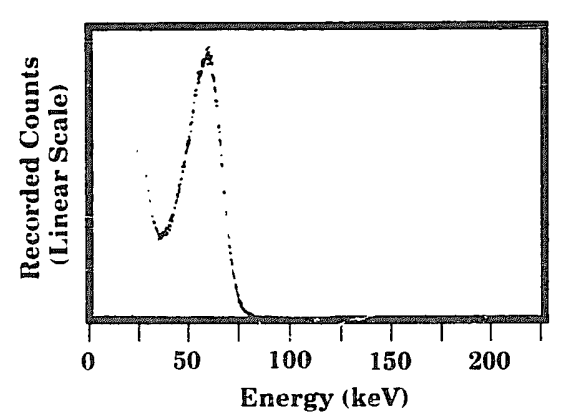

Fig. 8. Room temperature measurement of the differential pulse height spectrum exhibited by a $250 \mu \mathrm{m}$ thick bulk GaAs detector irradiated with $60 \mathrm{keV}$ gamma rays from ${ }^{2+1} \mathrm{Am}$. The detector resolution was measured to be $22 \mathrm{keV}(37 \%)$ at FWHM. 


\section{Conclusion}

A preliminary investigation of LEC bulk GaAs has indicated its promising use as room temperature operated radiation detector. Detectors of $100 \mu \mathrm{m}$ thick have demonstrated acceptable resolution for charged particle spectroscopy. Thicker devices would provide a very attractive room temperature X-ray detector provided that hole collection efficiency is improved. Material with a lower EL2 concentration will be utilized in future generation devices to reduce possible problems with deep donor ionization. Future Schottky based detectors will have epitaxial or implanted back side surfaces to reduce ohmic contact resistance and devices designed primarily for photon detection will incorporate highly doped $\mathrm{p}$ and $\mathrm{n}$ regions in order to fabricate $\mathrm{p}-\mathrm{i}-\mathrm{n}$ device structures.

\section{Acknowledgements}

The authors wish to express their gratitude to David Waechter, Alan Gibbs, and David Brown of Los Alamos National Laboratory, Victor P. Swenson and William N. Gibler of Texas A\&M University, and Ronald Rojeski, Jim McCartney, Jeff Fournier, and Jim Kulman of the University of Michigan for technical assistance in the fabrication and testing of the aforementioned devices. Proton implantation was performed by the Michigan Ion Beam Laboratory at the University of Michigan.

We also express gratitude towards our colleagues Dr. Jack East and Dr. Fred L. Terry, Jr. of the University of Michigan for their valuable collaboration with this project.

This project has been funded in part by Los Alamos National Laboratory subcontract 9-XGO-K7910-1.

\section{Appendiy:}

EL2 donors are typically present in LEC undoped Gafis at an approximate concentration of $10^{16} / \mathrm{cm}^{3}$ and appear in the bandgap at an energy of $0.81 \mathrm{eV}$ below the conduction band edge [7,12]. Assuming that the Fermi level of the undoped GaAs to be near midgap, the result is incomplete ionization of the EL2 donors at room temperature. Using Fermi-Dirac statistics in which the Fermi level of the undoped GaAs is approximately near midgap and the EL2 energy is $0.81 \mathrm{eV}$ below the conduction band edge [12], the total ionization of EL2 sites is on the order of $10^{14} / \mathrm{cm}^{3}$, which balances well with information from the supplier that the residual carbon acceptor concentration is on the order of $10^{14} / \mathrm{cm}^{3}$. Under the condition of applied reverse bias, the conduction band is moved up at the Schottky contact and the difference in energy between the Fermi level and the energy of the EL2 sites changes resulting in more ionization of the deep donors. From Poisson's equation we find:

$$
-\frac{\partial^{2} V}{\partial X^{2}}=\frac{\partial E}{\partial X}=\frac{q}{\epsilon_{\mathrm{b}}}\left(N_{\mathrm{d}}^{+}-N_{\mathrm{a}}^{-}-n_{0}\right) \text {, }
$$

where

$N_{\mathrm{d}}^{+}=N_{\mathrm{d}}\left(1-\frac{1}{1+\frac{1}{2} \mathrm{c}^{\left(t_{\mathrm{d}}-E_{\mathrm{i}}\right) / h T}}\right)$.

The difference between the Fermi energy and the donor energy changes with bias, and is therefore also a function of the voltage. The solution can be solved numerically to give an electric field distribution that has two significantly different regions. From the Schottky contact to a distance in the material at which complete ionization of the EL2 sites is realized, the electric field reduces with a constant slope equivalent to $q N_{\mathrm{d}} / \epsilon_{\mathrm{s}}$. Beyond the point of full ionization, the electric field distribution decays less rapidly to zero. Electron-hole pairs created by ionizing particles entering the high electric field region near the Schottky contact experience a much higher field strength than if created near the ohmic contact. The result is higher collection efficiency near the Schottky contact for holes. Since electrons reach a maximum velocity in GaAs near an electric field strength of $3 \times 10^{3} \mathrm{~V} / \mathrm{cm}$, the lower field strength near the ohmic contact poses less of a problem for electron collection. The low electric field present near the back side contact allows for some electron collection and may account for the small pulses observed from back side irradiation at low bias voltages. However, the very low field strength rear the ohmic contact translates to very inefficient hole collection for $\mathrm{e}-\mathrm{h}$ pairs produced in that region. The resulting inefficient charge collection would translate to poor energy resolution and lower pulse height. As the electric field is increased under higher voltages, carrier collection improves allowing for electrons and holes to be collected more efficiently.

\section{References}

[1] S.P. Swierowski and G.A. Armantrout. IEEE Trans. Nucl. Sci. NS-22 (Feb. 1975) 205.

[2] Guy A. Armantrout. S.P. Swierkowski, J.W. Sherohman and J.H. Yee. IEEE Trans. Nucl. Sci. NS-24 (Feb. 1977) 121.

[3] T. Kobayashi and S. Takayanagi. Nucl. Instr. and Meth. 44 (1966) 145.

[4] J.E. Eberhardt, R.D. Ryan and A.J. Tavendale. Nucl. Instr. and Meth. 94 (1971) 463. 
[5] R. Bertin. S. D'Auria, C. Del Papa, F. Fiori, B. Lisowski, V. O'Shea, P.G. Pelfer, K. Smith and A. Zichichi, Nucl. Instr. and Meth. A294 (1990) 211.

[6] G.M. Martin. J.P. Farges, G. Jacob and J.P. Hallais, J. Appl. Phys. 51 (1980) 2840.

[?] R.K. Willardson and A.C. Beer, Semiconductors and Semimetals, vol. 20 (Academic Press, Orlando, 1984).

[S] D.S. McGregor, R.D. White and M.H. Weichold. TEES Tech. Rept. Ser. (1985).
[9] D. Look, Electrical Characterization of GaAs Material and Devices (Wiley, New York, 198Y)).

[10] M. Shur, GaAs Devices and Circuits (Plenum, New York, 1987).

[11] J.F. Ziegler and J.P. Biersack, TRIM-90, Version 90.05 (1990).

[12] Y. Kitagawara, N. Noto, T. Takahashi and T. Takenaka, Appl. Phys. Lett. 48 (1986) 1664. 